\title{
Global precedence in visual pattern recognition
}

\author{
HOWARD C. HUGHES \\ Dartmouth College, Hanover, New Hampshire \\ W. MICHAEL LAYTON \\ AGS Corporation, Hanover, New Hampshire \\ and \\ JOHN C. BAIRD and LAURIE S. LESTER \\ Dartmouth College, Hanover, New Hampshire
}

\begin{abstract}
This paper describes the results of three experiments on the global precedence effect, using stimuli derived from the receptive field characteristics of neurons in the visual cortex. Thus, the local cue consists of oriented line segments whose sizes correspond to the average size of the central portion of receptive fields in parafoveal representations of the macaque primary visual cortex. The global cue comprises these line segments distributed within larger rectangular clusters. The first experiment showed that global precedence is obtained when the relative visibility of the local and global cues is specifically evaluated and found to be comparable. In the second experiment, variants of the Garner sorting task produced results indicating that global processing is largely independent of local cues, but that local processing depends heavily on the status of task-irrelevant global cues. These asymmetric interactions are consistent with the notion of a priority for processing global cues. The third experiment demonstrated that the mag. nitude of global precedence is inversely related to pattern luminance. This latter finding is interpreted, in the context of Sternberg's additive factors logic (1969), as indicating that this priority for global feature processing is at least partially attributable to aspects of early visual processes.
\end{abstract}

This paper is concerned with the way in which the visual system achieves globally organized percepts of pattern structure. There are two generally acknowledged ways in which this may occur. First, global pattern structure could be constructed from hierarchically organized processes that begin with a highresolution image analysis. Alternatively, the visual system could perform a parallel analysis of images at a variety of spatial scales (or spatial frequencies), and encode global pattern structure directly through a low-resolution scan. Traditionally, hierarchical approaches have been favored. A physiological implementation of such hierarchical systems was suggested by the work of Hubel and Wiesel $(1962,1965)$, and this model of image processing has had a great impact on conceptual approaches to pattern recognition. More recently, of course, a great deal of evidence which indicates that the visual system contains parallel channels tuned to different spatial scales (or spatial frequencies) has accumulated. Evidence in

Address all correspondence and requests for reprints to the first author, Department of Psychology, Dartmouth College, Hanover, NH 03755. This research was supported in part by a fellowship from the Alfred P. Sloan Foundation and in part by a Faculty Research award from Dartmouth College. We also wish to thank I. Golding for assistance in data collection, M. Fanselow and $\mathbf{L}$. Tassinary for assistance in the data analysis, and C. Fowler and $T$. Tighe for helpful criticisms of the manuscript. support of these parallel channels has come from both psychophysical (e.g., Ginsberg, 1977; Graham, 1981) and physiological studies (e.g., Stone, Dreher, \& Leventhal, 1979).

Recently, beginning with the experiments of Navon (1977), evidence which indicates that the global aspects of visual patterns are actually responded to more quickly than local pattern microstructure has begun to accumulate. This is what Navon called the "global precedence effect." The interpretation that such results indicate a global-to-local processing sequence has been questioned on several grounds, however. Garner (1983) has argued that many of the experiments on global precedence suffer from a shortcoming that complicates their interpretation; isolated reaction times to the local and global cues either have not been specifically evaluated or, when they have, have frequently not been equated. This difference quite possibly reflects a greater discriminability of the global cues, which would obviously bias the results in favor of global processing. Secondly, both Miller (1981) and Boer and Keuss (1982) have presented data they interpret as indicating that global precedence is not a perceptual effect, but rather is a manifestation of postperceptual processes. Both an attentional bias (Miller, 1981) and response competition (Boer \& Keuss, 1982) have been suggested as possible mechanisms for the effect. In addition, work 
on global precedence has tended to use "compound letter" stimuli, and very few other types of patterns have been explored (an exception can be found in Boer \& Keuss, 1982). In view of the possible implications that global precedence could have to visual pattern recognition, it would seem appropriate to explore the phenomenon using a wider range of stimulus materials.

The present series of experiments was designed to assess the relative speed of processing local and global cues which are generally felt to represent visual features in the most elementary sense; they are expected to be effective stimuli for orientation-selective cells in the visual cortex. Thus, the local cue consists of oriented line segments whose sizes correspond to the average size of the excitatory regions of foveal and parafoveal receptive fields in the primary visual cortex (Hubel \& Wiesel, 1974). These local features are confined to larger rectangular clusters, a configuration which represents the global cue. Our working hypothesis is that the global cue could, via spatial integration of the local features, directly activate cells whose receptive field dimensions correspond to the size of the rectangular clusters; that is, cells with larger receptive fields might integrate the local cues into a "global feature."

The global precedence effect obtained with these stimuli was robust. The first experiment provides a test of the idea that unequal visibility of the local and global cues may be a necessary condition for obtaining global precedence. The second experiment explores the asymmetric interactions between local and global spatial scales in the context of Garner's notion of dimensional integrality. The third experiment evaluates the effects of pattern luminance on the relative speed of processing local and global cues. The results suggest that global precedence may originate within the visual system, but that later processes can serve to modulate its magnitude.

\section{EXPERIMENT 1}

The goal of this experiment was to determine whether global precedence would appear when using the type of stimulus shown in Figure 1b. The discriminability of the local and global orientation cues was independently evaluated using the control patterns, examples of which are shown in Figures $1 \mathrm{c}$ and $1 \mathrm{~d} .{ }^{1}$ Thus, the experiment addresses the possibility that unequal discriminability of the cues represents a possible artifact in previous studies of global and local pattern processing.

\section{Method}

Subjects. Twenty introductory psychology students at Dartmouth College served as subjects. All subjects claimed to be emmetropic or were optically corrected.

Stimuli and Procedures. The task required that subjects rapidly classify patterns like those illustrated in Figures 1b-1d according
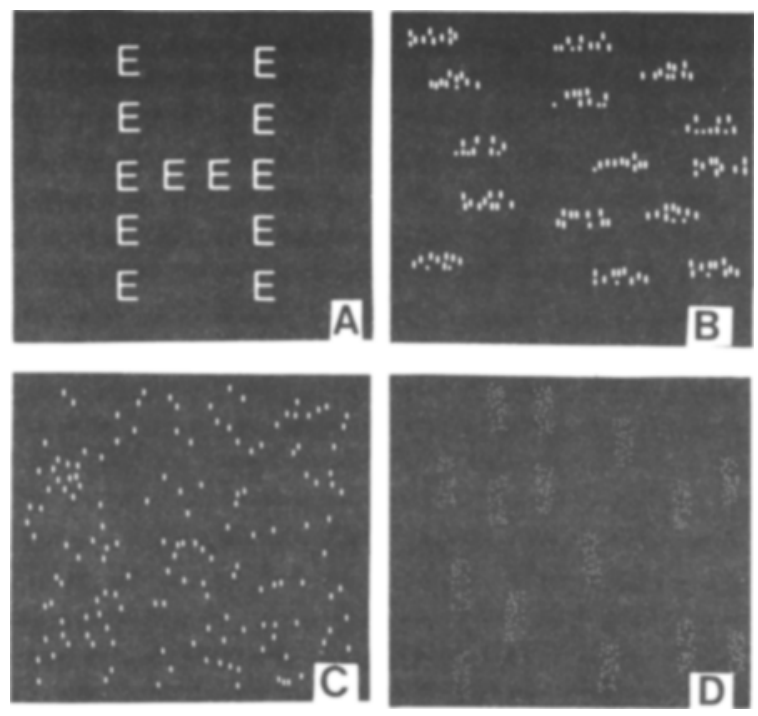

Figure 1. Examples of stimuli used in experiments on the global precedence effect. Figure 1a is an example of the type of stimulus commonly used; the stimuli in panels $b, c$, and $d$ were used in the present experiments. Each pattern in panels $b, c$, and $d$ contains 15 clusters and/or 150 line segments.

to their orientation. Figure $1 \mathrm{~b}$ has orientations that can be specified for either the global (cluster orientation) or the local (line orientation) spatial domain. Global precedence therefore predicts that cluster orientation should be judged more rapidly than line orientation, even though the lines should represent elementary visual features in the sense that they should be effective stimuli for neurons in the primary visual cortex. The single-dimension control stimuli illustrated in Figures $1 c$ and $1 \mathrm{~d}$ were designed to evaluate reaction times to the local and global cues independently. The pattern in Figure 1c provides no global cue (the lines are positioned randomly throughout the screen) but the same local cue as the "compound" pattern in Figure lb; the reverse is true of the global control pattern (Figure 1d). All three pattern types are equated for the number of pixels in the display, so the spaceaveraged luminance of each pattern is the same. Furthermore, the space-averaged luminance of the clusters is the same regardless of whether they are made from lines or dots. The luminance of each pixel was $.5 \mathrm{fL}$, and the background luminance was $.2 \mathrm{fL}$. These stimuli were computer generated and were constructed so that the position of both the clusters and the local pattern elements varied randomly from trial to trial, the only constraint being that individual pattern elements could not be closer than $.095 \mathrm{deg}$ and that adjacent clusters could not be closer than 1.44 deg of visual angle. The overall size of the stimulus field was $17.1 \times 16.3$ degrees, which is much greater than most estimates of the bandwidth of the lowest resolution channels in the human visual system (e.g., Kinchla \& Wolf, 1979; Wilson \& Bergen, 1979). The cluster dimensions were $2.9 \times .96 \mathrm{deg}$, and the line segments were $.45 \times .1 \mathrm{deg}$. These latter dimensions do correspond with estimates of two different size-specific channels (Wilson \& Bergen, 1979).

The patterns were displayed on a 12-in. video raster scan monitor for $100 \mathrm{msec}$ (onset/off set time of $16 \mathrm{msec}$ ). The subjects viewed the monitor from a distance of $60 \mathrm{~cm}$. They were instructed to fixate a small white dot at the center of the screen, and to depress one of two microswitches as quickly and accurately as possible, depending on whether a given pattern was classified as "horizontal" or "vertical." The microswitches were depressed with the index fingers of either hand, and the mapping of the two orientations to the preferred and nonpreferred hands was counterbalanced across subjects. 
A $1000-\mathrm{Hz}$ warning tone preceded the presentation of each pattern by $500 \mathrm{msec}$. Control of the experiment and data collection were under computer control.

Data were obtained in four separate experimental sessions. In the first, the subjects were presented with four series of patterns like that of Figure $1 \mathrm{~b}$, with each series containing a randomized sequence of 24 patterns in which line and cluster orientation were orthogonally combined. The four possible combinations of line and cluster orientation always occurred with equal frequency. Half the subjects were assigned randomly to one group and instructed to classify the patterns according to line orientation (Group 1); the other subjects were instructed to classify the same patterns according to cluster orientation (Group 2). Forty-eight practice trials were followed by $\mathbf{4 8}$ test trials. Reaction times to the control patterns were obtained from all subjects during three subsequent sessions. As in the experimental sessions, 48 practice trials were followed by 48 test trials and each session used 96 different patterns. Reaction times to these control patterns were evaluated under two conditions. In the first, local and global control patterns were presented during two separate sessions (single-cue control condition); in the second, both the local and global control patterns were intermixed within the same session (two-cue control condition). This second condition was included so that the number of possible stimulus alternatives was the same as in the experimental condition, and to permit an analysis of the effects of local and global cue shifts over successive trials. The testing sequence for the control conditions was the single-cue control for the previously relevant cue in the first condition, followed by the mixedcue control, followed by the single-cue control for the previously irrelevant cue.

\section{Results}

Errors. Error rates were low, averaging $4.58 \%$ across all conditions with a standard deviation of $1.34 \%$. The proportions of errors from each condition were transformed, using the arcsine transformation, and then analyzed by analysis of variance. This analysis revealed no significant differences in error rates, either between groups or between patterns, and no significant interaction. Thus, there is no evidence that speed-accuracy tradeoffs contributed to the results reported below.

Latency data. The principle results are illustrated in Figure 2, in which local responses are indicated by pluses and global responses are indicated by squares. Analysis of variance revealed significant main effects for both groups and pattern and a significant interaction $[F(4,72)=4.80, p<.002]$. Pairwise comparisons between individual group means were tested using the Newman-Keuls procedure. All reported differences between these means are significant with alpha $<.05$. These pairwise comparisons confirmed that reaction times to the experimental patterns are significantly slower when local orientation is the relevant cue, despite the fact that latencies in the singlecue control conditions did not differ, either within or between groups. There were also no within-group differences in latency to the local and global control patterns when they were intermixed, although intermixing did significantly slow' responses to the control patterns in the judge-local group. While a similar slowing is evidenced in the judge-global group, this latter difference was not reliable. Thus, comparisons among the various control conditions provides ample

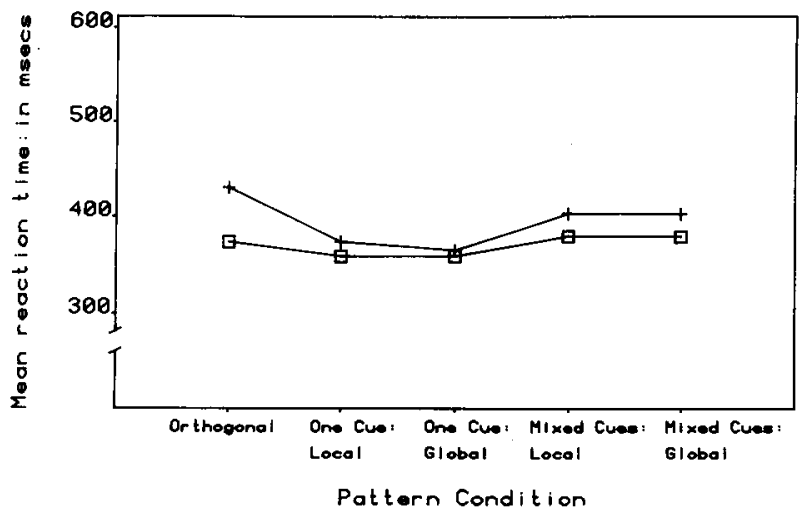

Figure 2. Group mean reaction times to classify the patterns shown in Figures 1b, 1c, and 1d according to orientation. Local judgments are indicated by pluses, and global judgments are indicated by squares.

evidence that these local and global cues are processed at equivalent rates when presented in isolation. However, when presented together, local processing is slowed, while global processing is not significantly affected. That is, within-group comparisons between the experimental and either type of control pattern shows significant differences for local but not for global judgments. Global precedence is thus obtained when the discriminability of both cues is equivalent. ${ }^{2}$

Effects of congruency between local and global orientation. A separate analysis was performed to determine the effect of the level of the irrelevant cue on classification speed. We use the terms congruent to refer to stimuli in which the relevant and irrelevant cues have identical orientations, and incongruent to refer to stimuli in which the two orientations differ. The data on the clustered line segments was sorted according to congruency, and the results are provided in Table 1 . A $2 \times 2$ analysis of variance (congruency $\times$ relevant cue) revealed significant main effects for both congruency and relevant cue, and a significant interaction (all ps $<.001$ ).

Comparisons between these means, using the Scheffé procedure, revealed significant congruency effects for both local and global orientation judgments (ps < .01). Importantly, global responses to incongruent patterns were significantly faster than local responses to congruent patterns $(p<.005)$. While congruency (which we assume reflects competing response tendencies) clearly influences the speed of these orientation judgments, these data emphasize

Table 1

The Effect of Congruency of the Irrelevant Cue on the Speed of Local and Global Orientation Judgments

\begin{tabular}{lcc}
\hline & Global RTs & Local RTs \\
\hline Congruent & $356.7 \pm 24.3$ & $407.9 \pm 27.0$ \\
Incongruent & $385.4 \pm 36.1$ & $454.6 \pm 36.9$ \\
\hline
\end{tabular}


that the magnitude of this effect is asymmetric, and that global judgments to incongruent patterns are faster than local judgments to congruent patterns, for which response competition should not be a factor.

Sequence effects in the two-cue control condition. The intermixing of local and global control patterns provided an opportunity to study the effects of cue changes over successive trials. These effects have also been the subject of a recent study by Ward (1982). The data were sorted according to the sequence of the cues presented on successive trials. As indicated in Table 2, this sequence effect was symmetric (localto-global and global-to-local changes increase RTs by the same amount, approximately $25 \mathrm{msec}$ ). The analysis confirmed that cue changes over successive trials significantly slowed responses to both the local and global control patterns $[F(3,54)=11.92, p<.0001]$. There were no between-group differences and no significant interactions. Finally, we examined these data for sequence effects when there was a change in orientation within a given spatial scale (e.g., local-horizontal to local-vertical). An analysis of variance on these data yielded no significant differences; these sequence effects are specific to changes in spatial scale.

Reaction times as a function of the foveal proximity of the local cue. One might expect that the distance of the local line segments from fixation would be greater when those lines were confined to clusters than when they were randomly distributed throughout the screen. Since visual acuity falls with increasing eccentricity, this could potentially render our comparison between line orientation judgments in the control and experimental patterns untenable. That is, one could argue that line orientation is less visible when the lines are confined to clusters simply because they are further from fixation there than they are in the local control patterns. We therefore computed both the minimum distance of any pixel to the fixation mark and the average distance of all the image points from fixation. These measures were obtained for all the local control and experimental patterns used in the experiment. The average minimum distance for all the control patterns was .63 deg, and the average for all the points was $5.9 \mathrm{deg}$. The corresponding figures for the two-dimensional experimental patterns were .76 and $5.7 \mathrm{deg}$. These differences seemed negligible. However, in order to be

Table 2

Sequence Effects in the Mixed-Cue Control Condition

\begin{tabular}{cccc}
\hline \multicolumn{4}{c}{ Pattern Sequence } \\
\hline Global-Local & Local-Global & Global-Global & Local-Local \\
\hline $400.5 \pm 45.4$ & $405.1 \pm 46.5$ & $378.9 \pm 32.9$ & $378.5 \pm 37.4$ \\
\hline
\end{tabular}

Note-Since there were no between-group differences, the data from both groups have been pooled. The data represents mean reaction times on the second trial for each of the indicated sequences. The intertrial interval was approximately 4 sec. sure that foveal proximity was not a factor, we analyzed the data from the judge-local group using only those local control patterns. (randomly distributed lines) in which the nearest line segment was at least $1.0 \mathrm{deg}$ from fixation and compared those reaction times to experimental patterns (clustered lines) in which the nearest line was no further than $.5 \mathrm{deg}$ from fixation. The group mean reaction times for this subset of patterns were: local control $=370.5$, congruent experimental patterns $=407$, and incongruent experimental patterns $=440[F(2,18)=9.12$, $\mathrm{p}<.002]$. Post hoc comparisons indicated that reaction times to all three pattern types differed significantly from each other. Thus, reaction times to the control patterns are faster than reaction times to the congruent experimental patterns, even when the nearest element in the control patterns is twice as far from fixation than the nearest element in the congruent experimental patterns.

\section{Discussion}

The results of this experiment clearly demonstrate global precedence, even though the control conditions indicated that the isolated local and global cues were equally discriminable (at least in terms of their choice reaction times). Since reaction times in the single-cue control condition produced comparable reaction times, it seems reasonable to conclude that, in this condition, visual processing rates for local and global cues are nearly the same. When compared with these control conditions, global precedence appears as a differential slowing of responses to local cues relative to global cues. It has previously been suggested that either attentional effects (Miller, 1981; Ward, 1982) or response competition (Boer \& Keuss, 1982) could produce this slowing of local responses in the presence of global cues. We next explored the results in this context.

The effects of attention. The data from the mixedcue condition indicate that when subjects are uncertain about which cue will be presented, reactions to both cues are slowed by equivalent amounts (about $25 \mathrm{msec}$ ). The analysis of the sequence effects shows that most of this slowing is attributable to the effect of shifting cues over successive trials, since when the orientation cue remained at the same spatial scale over successive trials, response latencies were about the same as in the single-cue conditions. This sequence effect is most likely attributable to an attentional mechanism that remains focused on the channel that was activated by the previous pattern, and similar mechanisms have been postulated to account for sequence effects in other situations (Luce, Baird, Green, \& Smith, 1980). Regardless of its mechanism, however, it is clear that subjects can direct their attention to either the local or global spatial scale with equal facility, since the magnitude of the sequence effect is symmetric. This symmetry in the allocation 
of attention to local and global cues would appear to be inconsistent with the notion that global precedence can be explained in terms of an attentional bias that favors the processing of global cues (Miller, 1981). Since Ward (1982) has reported essentially symmetric sequence effects using compound letter stimuli, it appears unlikely that the present experiment failed to reveal a bias because of the difference in stimuli used.

Attention to a specific location within the visual field (see Posner, Snyder, \& Davidson, 1980) may also have relevance to some studies of global precedence. For example, if a local cue is always presented in a given location, then attending to that location could selectively benefit local responses (see, e.g., Grice, Canham, \& Boroughs, 1983; Pomerantz \& Sager, 1975). Indeed, Grice et al. (1983) suggested that foveal presentation of local cues was necessary to insure adequate encoding of local cues, but the present data clearly indicate that responses to isolated local cues can be quicker than responses to local cues in compound patterns independent of retinal edcentricity. It appears that when spatial uncertainty is present, global precedence is the rule, whereas local precedence can be obtained if subjects have prior knowledge concerning the location of the local cues.

The effects of response competition. Aspects of the data also bear on the role of competing response tendencies in global precedence. Since global reactions to incongruent patterns were significantly faster than local responses to congruent patterns, the interference generated by response competition is not sufficiently great to provide a general explanation of why global responses are faster than local responses. The asymmetric magnitude of response competition may be interpreted as indicating that global cues have achieved their precedence prior to response selection, and that asymmetric response competition is a manifestation of global precedence rather than its cause.

\section{EXPERIMENT 2}

This experiment explored the relative speed of local and global reactions to the stimuli used in Experiment 1 , using a variant of Garner's sorting task. This task has previously been used to explore issues relating to serial versus parallel processing of twodimensional stimuli (Clark \& Brownell, 1976; Garner \& Felfoldy, 1970; Wood, 1974).

We were particularly concerned here with estimating the salience or availability of the local cues when presented in conjunction with global orientation cues. For example, should we expect to find a difference in sorting times of global cues when the irrelevant local cues are either negatively or positively correlated with the relevant global cue? If, when presented together, local cues were processed at essentially the same rate as global cues, then we might ex- pect to see substantial differences in global response times, depending on whether the local cues were positively or negatively correlated. Furthermore, under the assumption that an attentional bias was responsible for the general finding of global precedence, how might local response times be affected if the irrelevant global cue remained fixed over the course of an experimental session? On the assumption that an invariant global cue would be easier to ignore than one that varied, one might reasonably expect that, in this situation, local response times might approximate global RTs. In view of the finding, from the first experiment, that, with single-dimension stimuli, attention could be effectively allocated to either local or global cues, one might expect that such a condition might provide an estimate of local processing rates in the presence of global cues when the contribution of attentional variables had been minimized.

We therefore examined the speed of classification responses to local and global orientation cues under the following conditions: (1) orthogonal, independent combination of line and cluster orientation; (2) positive correlation, lines and clusters always in the same orientation, which varies randomly across trials; (3) negative correlation, lines and clusters always in different orientations, which varies across trials; (4) unidimensional variation, variation of the relevant cue while the irrelevant cue remains fixed in one orientation; and (5) single-dimension controls, the control conditions from Experiment 1 were also included in Experiment 2.

\section{Method}

Subjects. Twenty-four undergraduates at Dartmouth served as subjects. As in Experiment 1, all claimed to have normal acuity or were optically corrected.

Stimuli and Procedures. The stimuli were the same as those used in Experiment 1, with the above noted differences in the possible combinations of local and global orientation cues. The subjects were assigned randomly to either a judge-local or a judge-global group. Each subject participated in all four experimental conditions and in the single-cue control conditions. Each condition was run in a daily session, and the order was randomized across subjects, with the exception that the control patterns were always presented in the fifth (last) session.

Prior to the first session, subjects were given 48 practice trials followed by 48 test trials. In subsequent sessions, 24 practice trials were given. Practice was always on the stimulus subsets used in that particular session. Other aspects of the procedure were the same as in Experiment 1.

\section{Results}

Errors. Error rates were transformed, using the arcsine transformation, and then analyzed by analysis of variance. The greatest number of errors occurred in the orthogonal and negatively correlated conditions $(6.8 \%$ and $6.7 \%$, respectively), and the lowest error rates occurred in the local and global control conditions $(2.4 \%$ and $2.8 \%$, respectively). The error analysis revealed a significant main effect of pattern condition $[F(5,110)=5.603, p<.0001]$, but no other 
differences were found. Post hoc comparisons between the six pattern conditions revealed that the orthogonal, negatively correlated, and unidimensional conditions produced more errors than the other conditions (positive correlation and local and global control conditions). Although the interaction between groups and pattern condition was not significant, the judge-local group made more errors in every condition except the unidimensional variation and the local control conditions. Since those conditions that produce higher error rates also produced the slowest reaction times, there is no indication that speed-accuracy tradeoffs contaminated the results.

Latency data. The major reaction time results are illustrated in Figure 3. These data were analyzed by analysis of variance, and subsequent pairwise comparisons were made using the Newman-Keuls procedure. The analysis indicated a significant betweengroups effect $[F(1,22)=4.32, p<.05]$, a significant effect of pattern condition $[F(5,110)=22.7, p<.0001]$, and a significant interaction $[F(5,110)=9.22, p<$ $.0001]$. The results from the orthogonal and the two single-dimension control tasks replicate the principle effect of Experiment 1; that is, global precedence was obtained using equally discriminable local and global cues.

There was no evidence for redundancy gains when cluster orientation was the relevant cue. In fact, none of the two-dimensional conditions differed from one another for globally directed responses, but the orthogonal and the negatively correlated tasks were slower than the single-dimension control task.
Between-group comparisons across all four twodimensional conditions confirm that locally directed orientation judgments were slower than global judgments in every condition except the positively correlated condition. Comparisons between the twodimensional patterns indicated that when local orientation was relevant, RTs in the orthogonal and negatively correlated conditions did not differ, but were slower than RTs in the unidimensional variation and the positively correlated conditions. The unidimensional condition was significantly slower than the positively correlated task.

While it is true that local RTs were equivalent to global RTs when the global cue was always in the same orientation as the local, it is not clear that this finding represents a true redundancy gain, because there is a strong possibility that subjects were basing their reactions on the global rather than the local cue.

Congruency effects. The effect of local and global congruency were evaluated in the two conditions in which this factor was relevant-the orthogonal and the unidimensional conditions. Figure 4 summarizes these effects, which were analyzed in a separate ANOVA. The analysis indicated a significant main effect of congruency $[F(1,22)=15.35, p<.001]$ and groups $[F(1,22)=16.25, p<.001]$ and a marginal effect of pattern type [orthogonal vs. unidimensional, $F(1,22)=3.87, p<.06]$. In addition, there were interactions between congruency and groups $(p<.001)$ and a triple interaction between congruency, pattern condition, and groups $(p<.01)$. Post hoc comparisons showed that congruency affected local judg-

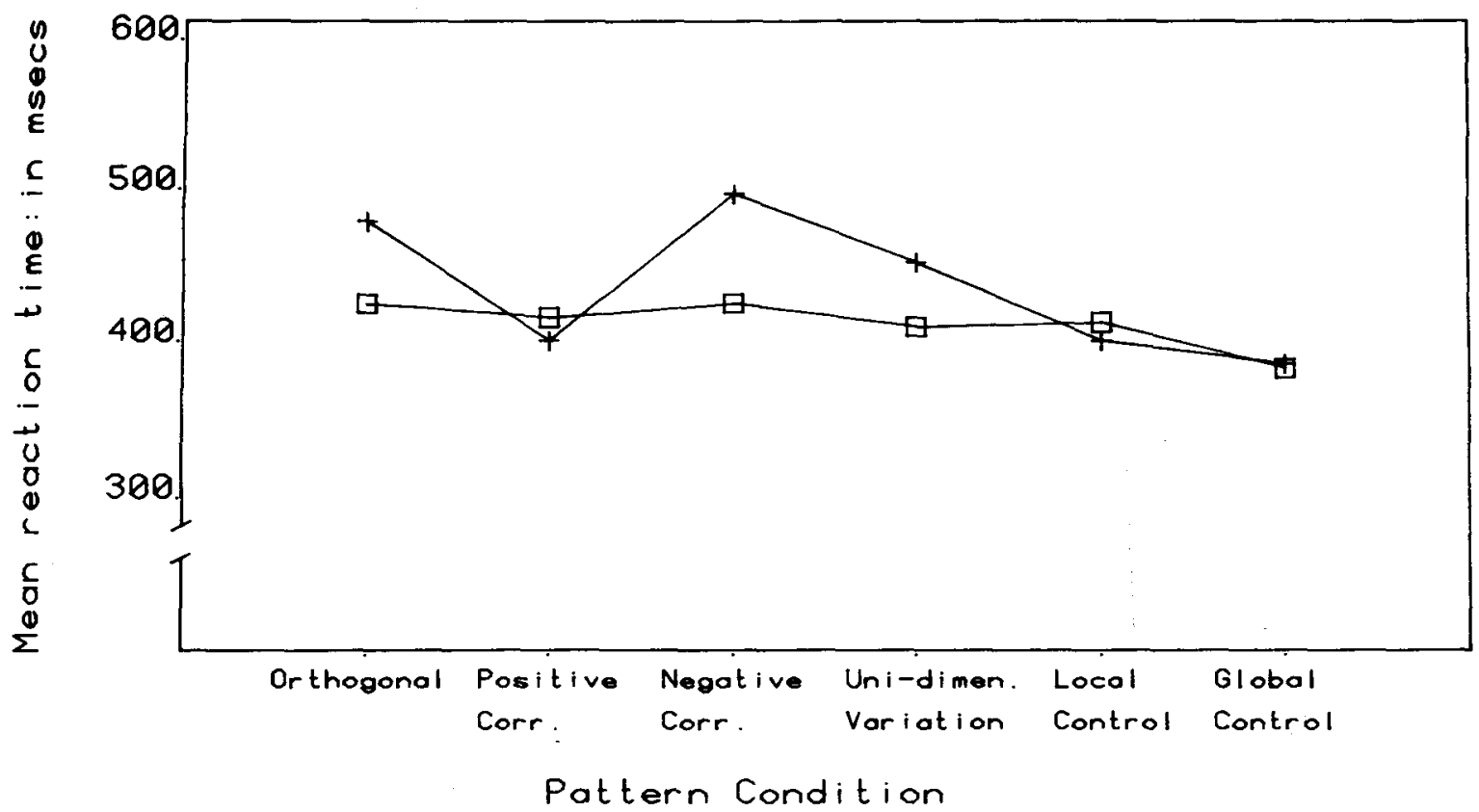

Figure 3. Summary of the data from Experiment 2. Pluses represent local judgments, and squares represent global judgments. 


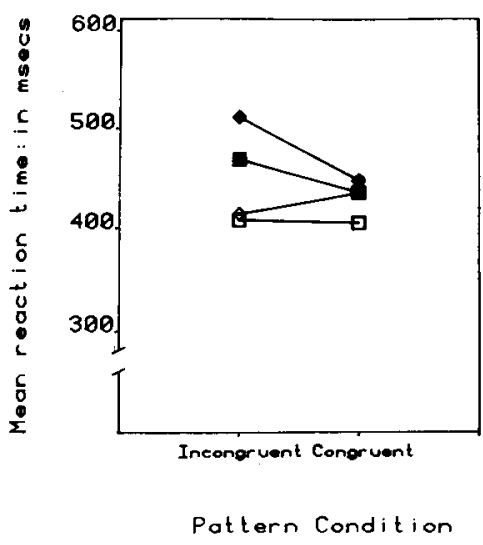

Figure 4. Congruency effects from Experiment 2. Diamonds represent data from the orthogonal condition, and squares represent data from the unidimensional variation condition. Filled symbols denote local responses, and open symbols denote global responses.

ments in both conditions but, in contrast to the first experiment, did not influence global judgments. As in Experiment 1, the magnitude of these congruency effects appears insufficient to account for the obtained differences in local and global RTs, inasmuch as local judgments to congruent patterns were consistently slower than global judgments to incongruent patterns. While there appears to be an "inverted" congruency effect for global judgments in the orthogonal condition (congruent RTs were slower than incongruent RTs), this effect was not reliable (although it just missed reaching the .05 level of significance). In view of the results from the same condition in Experiments 1 and 3, this aspect of the data is clearly anomalous. In Experiment 1, we obtained a modest benefit of congruency for global judgments, but in Experiment 3 there was no benefit. Thus, the congruency effect when the relevant cue is at the global scale is apparently very weak, and it may not be that surprising to occasionally see the effect go in the other direction.

\section{Discussion}

The unidimensional variation task represents an attempt to minimize the distracting effects of random variation of the global cue on local reactions. Since local responses under this condition were significantly faster than those obtained in the orthogonal and the negatively correlated conditions, it seems clear that the procedure was at least partially effective. The implication is that attentional factors can enhance the magnitude of global precedence. It is still true, however, that local reactions in this task were slower than global reactions in the orthogonal condition. In fact, local reactions to congruent patterns in the unidimensional variation condition were slower than global responses to incongruent patterns in the orthogonal condition $(p<.05)$. Thus, when the com- bined influences of response competition and distraction by irrelevant global cues are minimized, local RTs are still slower than global RTs generated under conditions in which these factors are presumably most potent. It would appear that neither response competition nor attentional biases can entirely account for the general finding of global precedence, although it is clear that both of these factors can enhance the magnitude of global precedence.

Local responses were equivalent to global responses when the two cues were positively correlated, but we are reluctant to regard this as evidence that, in this condition, local processing is as fast as global processing, because the subjects could have easily been responding to the global cue. Several previous reports have considered this possibility in what has been called the "selective serial processing" strategy (Biederman \& Checkosky, 1970; Felfoldy \& Garner, 1971; Garner \& Felfoldy, 1970; Wood, 1974). According to this idea, subjects can generate apparent redundancy gains for positively correlated dimensions in the absence of true parallel processing if one of the dimensions produces faster reaction times than the other in a selective attention task. Since this condition is clearly met in the present experiment, it is most parsimonious to attribute the increased sorting times for nominally local cues to this "cue-shifting" strategy, as it is the only case showing any evidence that local responses can be generated as quickly as global responses. Possibly, had some "catch trials" (incongruent patterns) been included in the session to insure that the subjects were indeed responding to the local cue, response times would have been slowed.

Thus, the only evidence that local responses can approximate the speed of global responses comes in a situation in which the subjects are probably responding to the global cue.

\section{EXPERIMENT 3}

The first two experiments indicate that neither response competition nor attentional factors are sufficiently powerful to provide a general explanation of global precedence. Because global precedence is obtained even when these factors operate in favor of local processing, we felt it appropriate to reconsider the possibility that global precedence had its origins within the visual system. Experiment 3 was intended to directly evaluate this suggestion. The experiment represents an application of Sternberg's additive-factors method for associating task variables with particular processing stages. According to this analysis, variables that have interactive effects on reaction times influence the same processing stage, whereas additive effects suggest that the variables influence different processing stages (Sternberg, 1969). Sanders (1977) has summarized the evidence that Sternberg's method generates a consistent and intuitively valid account 
of the influence of a wide variety of task variables on different stages of the reaction process. The present experiment was designed to determine whether variation in stimulus luminance (a variable which clearly influences "stimulus encoding") has additive or interactive effects on the size of the global precedence effect.

\section{Method}

Subjects. Sixteen undergraduates at Dartmouth served as subjects. All subjects had normal acuity or were optically corrected.

Stimuli and Procedures. The stimuli were the same as in Experiment 1 . The video screen was viewed through neutral density filters which allowed luminance to be varied over a range of $3 \mathrm{log}$ units. Luminance was reduced by using $0,1,2$, and $3 \log$ unit density filters. As in the previous experiments, the subjects were assigned randomly to either a judge-local or a judge-global group, and each was tested on the experimental and control patterns at each luminance level. All subjects first responded to the experimental patterns and then to the control patterns, the latter being presented in a counterbalanced order. Within a given pattern condition, the four luminance levels were presented according to a Latin square. All other aspects of the experiment were the same as in the previous experiments.

\section{Results}

Errors. The percentage of incorrect responses over the entire experiment was $5.7 \%$. The rate for local judgments was $6.7 \%$, while that for global judgments was $4.8 \%$. As before, the error rates were transformed using the arcsine and three-factor analysis of variance (luminance $\times$ pattern $\times$ groups). This analysis revealed significant main effects of luminance $[F(3,42)$ $=4.46, \mathrm{p}<.01]$ and a marginally significant effect of groups $[F(1,14)=4.1, p<.06]$. Post hoc comparisons between the four means contributing to the luminance effect showed that only the second and fourth luminance level differed from one another. No other differences in error rates were found.

Latency data. The latency data were also analyzed by analysis of variance, and are summarized in Figure 5. As indicated in Figure 5, there were significant main effects for pattern and luminance but not for groups. In addition, there was a two-way interaction between pattern and groups $[F(2,28)=23.35, p<$ .001 ], a marginal interaction between pattern and luminance $[F(6,84)=2.13, p<.06]$, and a triple interaction between pattern, groups, and luminance $[F(6,84)=2.62, p<.025]$. To evaluate whether the three-way interaction was attributable to growth in the size of global precedence with decreasing luminance rather than an interaction between luminance and RTs to the control patterns, we performed separate comparisons on the experimental and the control conditions, and the significance levels were corrected for the number of comparisons. This analysis revealed a significant interaction between local and global RTs in the experimental condition $[F(3,84)=6.26$, $\mathrm{p}<.02]$, but not in the control conditions $[\mathrm{F}(9,84)$ $=1.27, \mathrm{p}=.27]$. The differential effects of luminance are perhaps more clearly illustrated in Figure 6, which

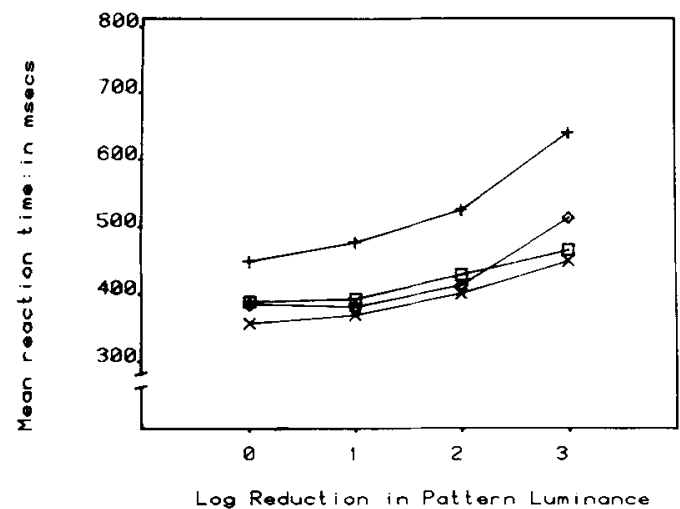

Figure 5. Mean reaction times for orientation judgments as a function of pattern luminance. Pluses represent local responses to "compound" patterns, and squares indicate global responses. The control data from both groups bave been pooled for clarity. Diamonds represent means for the local control condition, and Xs represent data from the global control condition.

presents the relevant comparisons as difference scores instead of the raw RT data. Thus, decreasing luminance enhanced the magnitude of global precedence without differentially affecting the visibility of the isolated local and global cues. It is well known that visual acuity declines with reductions in luminance, but the analysis indicates that acuity was not a factor in the present experiment. Inspection of Figure 6 indicates that at the fourth luminance level, local cues in the control condition may have been slightly less discriminable than the global control cues. Since the interaction between RTs in the control conditions and luminance was not significant, it is inappropriate to specifically evaluate the means at the dimmest luminance. It is worth noting, however, that comparisons of the local RTs in the experimental condition indicated that local judgments slowed significantly for all pairwise comparisons except those between the first and second luminance levels. In contrast, similar comparisons for the global RTs indicated that only the first and fourth luminance level differed from one another. Thus, there are clear indications that the rate of change in the RT-luminance curve is different for local and global judgments in

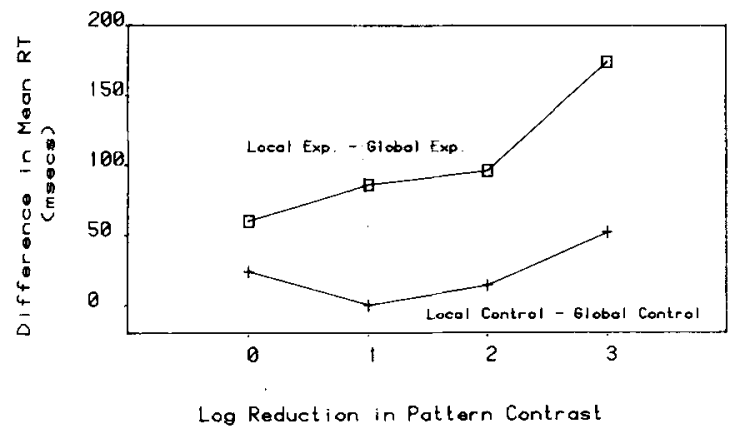

Figure 6. Effects of luminance on local and global reaction times expressed as difference scores. 
the experimental condition, and that this difference is not restricted to the dimmest luminance, where the local and global cues may differ slightly with respect to their isolated visibility.

Congruency effects. The effect of congruency between the relevant and irrelevant cue was evaluated over all four luminance levels by analysis of variance. These data are summarized in Figure 7. The analysis indicated significant main effects of congruency $[F(1,14)=7.69, p<.02]$, luminance $[F(3,42)=21.96$, $\mathrm{p}<.001]$, and groups $[F(1,14)=12.69, \mathrm{p}<.01]$. In addition, the interaction terms for congruency and luminance $(p<.001)$, congruency and groups $(p<$ $.01)$, and luminance and groups $(p<.02)$ were all significant, as was the triple interaction between congruency, luminance, and groups $(\mathrm{p}<.001)$. Pairwise comparisons (Newman-Keuls) among the individual means confirmed significant congruency effects for local judgments for all but the lowest luminance, but there were no significant congruency effects for global judgments.

\section{Discussion}

The results of Experiment 3 clearly indicate that the magnitude of global precedence is dependent on the luminance of the patterns. Global precedence grew in strength as luminance was reduced, and this change occurred despite the fact that the visibility of the isolated local and global cues remained comparable over this range of stimulus luminances. The effects of congruency on local judgments initially grew with reductions in pattern luminance, but this effect completely dissipated at the lowest luminance level. This loss of the congruency effect was not anticipated, and we have no ready explanation for it. In some sense, it presumably represents a boundary condition for response competition. For example, it might be suggested that when the global cues are identified sufficiently in advance of the local cues, congruency between the two no longer plays a role. Alternatively, the effects of competing response ten-

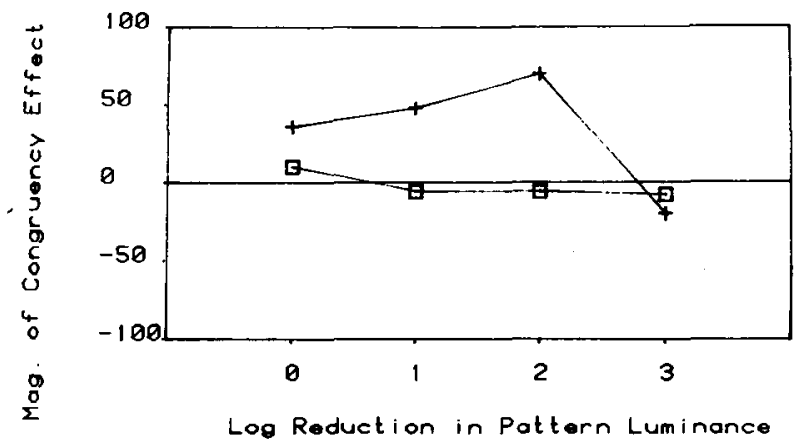

Figure 7. Congruency effects as a function of pattern luminance. For clarity, the ordinate indicates the difference between congruent and incongruent stimuli (incongruent-congruent) for local (plusses) and global (squares) orientation judgments. dencies may depend in a direct way on high levels of sensory activity, so when the activity falls with decreasing luminance, response competition dissipates. In any case, the effect of response competition (as revealed by these congruency effects) also depends on pattern luminance. These findings are difficult to explain if it is assumed that global precedence (as well as the asymmetric effects of response competition) is entirely a postperceptual effect (cf. Boer \& Keuss, 1982; Miller, 1981). They are, however, consistent with the suggestion that global precedence has its origins in early visual processing.

\section{GENERAL DISCUSSION}

The present series of experiments is interpreted as indicating that postperceptual factors cannot account for all aspects of global precedence. The results indicate that the effect is obtained even when the relative visibility of the local and global cues is specifically evaluated and found to be equivalent. In addition, it seems unlikely that response competition can be used to explain the effect, since local responses in the absence of response competition (i.e., to congruent patterns) are slower than global responses in the presence of response competition (incongruent patterns). Moreover, since the magnitude of the congruency effect grows (at least initially) with reductions in luminance, it would seem that the strength of competing response tendencies depends on sensory factors; that is, response competition may be more a manifestation of global precedence than its cause. Finally, our attempts to minimize the distracting influence of irrelevant global cues failed to produce local reactions which approximate the speed of global reactions, a finding that is difficult to reconcile with assertions that local information is processed as rapidly as global information (cf. Miller, 1981).

Our view of the essential elements of the global precedence effect that emerges from these and previous experiments is that (1) local and global cues seem to be processed in parallel (although not necessarily at the same rate; cf. Boer and Keuss, 1982; Miller, 1981), (2) this occurs even when isolated local and global cues are equally visible, and (3) global precedence derives from a slowing of local processing relative to the processing speed of the same cue presented in isolation, and this slowing is either absent or very weak when global cues are relevant. In addition, the magnitude of global precedence depends on stimulus luminance, and this dependence is also not an artifact of unequal visibility of the local and global cues. The luminance effect is particularly difficult to explain on the assumption that global precedence is entirely a postperceptual phenomenon. The results of the third experiment indicate that some aspect of the early processing of visual information may act to confer an advantage to the processing of 
global features. We are currently exploring a network model of global precedence which suggests the existence of asymmetric inhibitory interactions between parallel sensory channels tuned to the local and global spatial scales. The major assumption is that channels tuned to low-resolution processing inhibit highresolution channels more than the converse. It is interesting to note that recent physiological data provides some support for this hypothesis (Morrone, Burr, \& Maffei, 1982).

\section{REFERENCES}

Biederman, I., \& Checkosky, S. F. (1970). Processing redundant information. Journal of Experimental Psychology, 83, 486-490.

Boen, L. C., \& Keuss, P. J. G. (1982). Global precedence as a postperceptual effect: An analysis of speed-accuracy tradeoff functions. Perception \& Psychophysics, 31, 358-366.

Clark, H. H., \& Brownell, H. H. (1976). Position, direction and their perceptual integrality. Perception \& Psychophysics, 19, 328-334.

Felfoldy, G. L., \& Garner, W. R. (1971). The effects on speeded classification of implicit and explicit instructions regarding redundant dimensions. Perception \& Psychophysics, 9, 289-292.

Garner, W. R. (1983). Asymmetric interactions of stimulus dimensions in perceptual information processing. In $T$. Tighe (Ed.), Proceedings of the Conference on Perception and Cognition. Hillsdale, NJ: Erlbaum.

Garner, W. R., \& FElfoldy, G. L. (1970). Integrality of stimulus dimensions in various types of information processing. Cognitive Psychology, 1, 225-241.

GinsBerg, A. (1977). Visual information processing based on spatial filters constrained by biological data. Doctoral dissertation, University of Cambridge.

Graham, N. (1981). Psychophysics of spatial frequency channels. In M. Kubovy \& J. R. Pomerantz (Eds.), Perceptual organization (pp. 1-25). Hillsdale, NJ: Erlbaum.

Grice, G. R., Canham, L., \& Boroughs, J. M. (1983). Forest before trees? It depends on where you look. Perception \& Psychophysics, 33, 121-128.

Hubet, D. H., \& Wiesel, T. N. (1962). Receptive fields, binocular interaction and functional architecture in the cat's visual cortex. Journal of Physiology, 160, 106-154.

HubeL, D. H., \& Wiesel, T. N. (1965). Receptive fields and functional architecture in two non-striate visual areas (18 and 19) of the cat. Journal of Neurology, 28, 229-289.

HubeL. D. H., \& Wiese L, T. N. (1974). Uniformity of monkey striate cortex: A parallel relationship between field size, scatter, and magnification factor. Journal of Comparative Neurology, 158, 295-305.

Kinchla, R. A., \& WoLF, J. M. (1979). The order of visual processing: "Top-down," "bottom-up," or "middle-out." Perception \& Psychophysics, 25, 225-231.

Luce, R. D., Baird, J. C., Green, D. M., \& Smith, A. F. (1980). Two classes of models for magnitude estimation. Journal of Mathematical Psychology, 22, 121-148.

Millek, J. (1981). Global precedence in attention and decision. Journal of Experimental Psychology: Human Perception and Performance, 7, 1161-1174.

Morrone, M. C., Burr, D. C., \& Maffei, L. (1982). Functional implications of cross-orientation inhibition of cortical visual cells. I. Neurophysiological evidence. Proceedings of the Royal Society of London: Series B, 216, 335-354.

Navon, D. (1977). Forest before the trees: The precedence of global features in visual perception. Cognitive Psychology, 9, 353-383.
Pomerantz, J. R., \& Sager; L. C. (1975). Asymmetric integrality with dimensions of visual pattern. Perception \& Psychophysics, 18, 460-466.

Posner, M. I., Snyder, C. R. R., \& Davidson, B. J. (1980). Attention and the detection of signals. Journal of Experimental Psychology: General, 109, 160-174.

SANDERs, A. F. (1977). Structural and functional aspects of the reaction process. In S. Dornic (Ed.), Attention and performance $V I$ (pp. 3-25). Hillsdale, NJ: Erlbaum.

Sternaerg, S. (1969). The discovery of processing stages: Extensions of Donder's method. In W. G. Koster (Ed.), Attention and performance II (pp. 276-315). Amsterdam: North-Holland.

Stone, J., Dreher, B., \& Leventhal, A. (1979). Hierarchical and parallel mechanisms in the organization of the visual cortex. Brain Research Reviews, 1, 345-394.

WARD, L. M. (1982). Determinants of attention to local and global features of visual forms. Journal of Experimental Psychology: Human Perception and Performance, 8, 562-581.

Wilson, H. R., \& Bergen, J. R. (1979). A four mechanism model for threshold spatial vision. Vision Research, 19, 19-32.

Wood, C. C. (1974). Parallel processing of auditory and phonetic information in speech discrimination. Perception \& Psychophysics, 15, 501-508.

\section{NOTES}

1. The intent of these control patterns was to assess the relative visibility of the local and global cues when they appeared in isolation. For this reason, we chose a random spatial distribution. Based on the assumption that the local cues are encoded by a population of orientation-specific neurons whose receptive field sizes correspond to the sizes of the lines, a random distribution should selectively activate a subset of these neurons. We argue that this provides a relatively pure indication of their visibility. While other types of control patterns might be devised, a nonrandom distribution would create some kind of global pattern. This would undermine any attempt to evaluate local line orientation in the absence of a global pattern structure. In view of some recent evidence showing that the simultaneous presentation of two patterns at different spatial scales can generate powerful inhibitory effects on neurons that respond well to one component in isolation (Morrone, Burr, \& Maffei, 1982), we felt it important to use control patterns that provided information at only one spatial scale.

2. The local control patterns used in these experiments contained the same number of lines as the experimental patterns, although the spatial density of the lines was greater in the experimental patterns. It seemed possible that this difference in density could produce lateral interactions which might degrade the visibility of the lines when they were distributed within the clusters. Although we are indeed arguing that the visibility of the lines is influenced by their spatial distribution, we wanted to be sure that this influence was not related to the difference in density. We therefore ran a separate experiment in which 10 new subjects classified randomly distributed line segments according to their orientation. Two different pattern conditions were compared; the first presented the same number of lines as in the experimental patterns, and the second presented randomly distributed lines with the same average density as the experimental patterns. Mean reaction times for the low- and high-density patterns were 349.3 and $356.6 \mathrm{msec}$, respectively. Thus, there is no indication that differences in the density of the local features alter the conclusion that the local and global features are equally discriminable.

It might also be argued that line orientation judgments based on 150 randomly distributed line segments represents a texture discrimination, and that when the same number of lines are confined to clusters, the texture is disrupted, leading to a decreased discriminability and increased RTs. In order to evaluate this possibility, we obtained RTs to patterns that contained only 15 line segments (the same number as in the global control patterns). Five 
additional subjects were tested with these patterns, and their choice reaction times were compared with those obtained from our standard local control patterns. The mean for the 15 lines was $\mathbf{3 4 9 . 2}$ msec, and the mean for 150 lines was $340.2 \mathrm{msec}$. This difference was not reliable $[\mathrm{t}(4)=1.40, \mathrm{p}<.23$, one-tailed].

Although we regard the local control that equates the numerosity of local cues with that of the compound patterns as the most appropriate, it seems clear that when the local elements are randomly distributed, factors of uniformity and number make little difference. It is worth noting that when there are only 15 lines in the pattern, subjects are probably perceiving the orientation of the individual lines rather than some overall texture.

(Manuscript received November 1, 1983; revision accepted for publication March 9, 1984.) 